\title{
Towards Atomic Scale Quantum Structure Fabrication in 2D Materials
}

Ondrej Dyck ${ }^{1,2^{*}}$, Andrew Lupini ${ }^{1,2}$, Maxim Ziatdinov ${ }^{1,2,3},{\mathrm{Xin} \mathrm{Li}^{1,2} \text {, Sergei V. Kalinin }}^{1,2}$ and Stephen Jesse $^{1,2}$

1. Center for Nanophase Materials Sciences, Oak Ridge National Laboratory, Oak Ridge, TN, USA.

2. Institute for Functional Imaging of Materials, Oak Ridge National Laboratory, Oak Ridge, TN, USA.

3. Computational Sciences \& Engineering Division, Oak Ridge National Laboratory, Oak Ridge, TN, USA.

* Corresponding author: dyckoe@ornl.gov

Many theoretical studies have revealed the exceptional physical properties of $2 \mathrm{D}$ materials for device applications ranging from spin diodes [1] to qubits [2-5], quantum emitters [6], and sensors [7, 8]. The unique properties of these potential devices arise from the specific atomic structure theorized - where even slight deviations from the idealized structure can lead to a significant alteration of the desired property. Thus, exquisite control over the positioning of each atom within the device is, in many cases, not only desirable but imperative for device performance.

Studies using aberration-corrected scanning transmission electron microscopes (STEMs) as atomic manipulation platforms have demonstrated atomically-precise positioning of dopant atoms in a host matrix and highlight some of the near term challenges for advancing this field[9-14]. The atomic manipulation process involves an electron beam that can be localized on or adjacent to a single atom. Imaging in the STEM of intentional modifications requires additional beam exposure of the sample, which can produce unintended alterations or damage. In addition, some sample transformations can be driven at one beam energy (e.g., $100 \mathrm{keV}$ in graphene) whereas mitigating excess sample damage may require operating at a lower beam energy (e.g., $60 \mathrm{keV}$ ). Here we discuss new strategies employed to address these challenges and enable routine atom-by-atom manipulation.

One strategy that can be used to reduce unintended sample alterations is sparse scanning. In this case, we employ an inpainting technique [15] that operates in real time to reduce the electron dose via sparse sampling while maintaining the information content in the image. A second strategy is to operate the objective lens in a constant power mode to enable rapid changing of the accelerating voltage, which allows access to multiple beam energies in a single session. Figure 1 illustrates the use of this strategy to form a 10-atom-wide arm-chair graphene nanoribbon, in which a $\sim 1 \mathrm{eV}$ band gap is purported to emerge [16]. A third strategy is to leverage deep learning tools for defect detection, categorization, and evolution under various sample conditions (e.g. beam energy, temperature etc.) $[17,18]$. Finally, a specialized microscope interface is desired, which can quickly switch between imaging and manipulation modes, offers custom scan paths, real-time filtering, and allows the operator greater control and monitoring of the sample state during manipulation.

These advances are enabling a significant improvement in the number and types of defects that can be produced as well as producing statistical-level data on beam-sample and beam-defect interactions. Together these rich streams of data form the knowledge-base from which controllable manipulation events can be extracted [19]. 
References:

[1] Y-J Dong et al., Scientific Reports 4 (2014), p. 6157.

[2] R Patrik and T Björn, Nanotechnology 21 (2010), p. 302001.

[3] B Trauzettel et al., Nature Physics 3 (2007), p. 192.

[4] GY Wu and NY Lue, Physical Review B 86 (2012), p. 045456.

[5] F Léonard in "Physics of Carbon Nanotube Devices", (William Andrew).

[6] I Aharonovich and M Toth, Science 358 (2017), p. 170.

[7] M Di Ventra and M Taniguchi, Nature Nanotechnology 11 (2016), p. 117.

[8] SJ Heerema and C Dekker, Nature Nanotechnology 11 (2016), p. 127.

[9] O Dyck et al., Small 14 (2018), p. 1801771.

[10] O Dyck et al., Applied Physics Letters 111 (2017), p. 113104.

[11] O Dyck et al., Nano Research (2018).

[12] BM Hudak et al., ACS Nano (2018).

[13] T Susi, JC Meyer and J Kotakoski, Ultramicroscopy 180 (2017), p. 163.

[14] T Susi et al., ArXiv eprints (2018).

[15] X Li et al., arXiv preprint arXiv:1805.04957 (2018).

[16] S Dutta and SK Pati, Journal of Materials Chemistry 20 (2010), p. 8207.

[17] A Maksov et al., npj Computational Materials 5 (2019), p. 12.

[18] M Ziatdinov et al., ACS Nano 11 (2017), p. 12742.

[19] Work was supported by the Laboratory Directed Research and Development Program of Oak Ridge National Laboratory, managed by UT-Battelle, LLC for the U.S. Department of Energy (O.D., M.Z., X.L. S.J.), Oak Ridge National Laboratory's Center for Nanophase Materials Sciences (CNMS), a U.S. Department of Energy Office of Science User Facility (S.V.K.), and the U.S. Department of Energy, Office of Science, Basic Energy Sciences, Division of Materials Science and Engineering (A.R.L.).
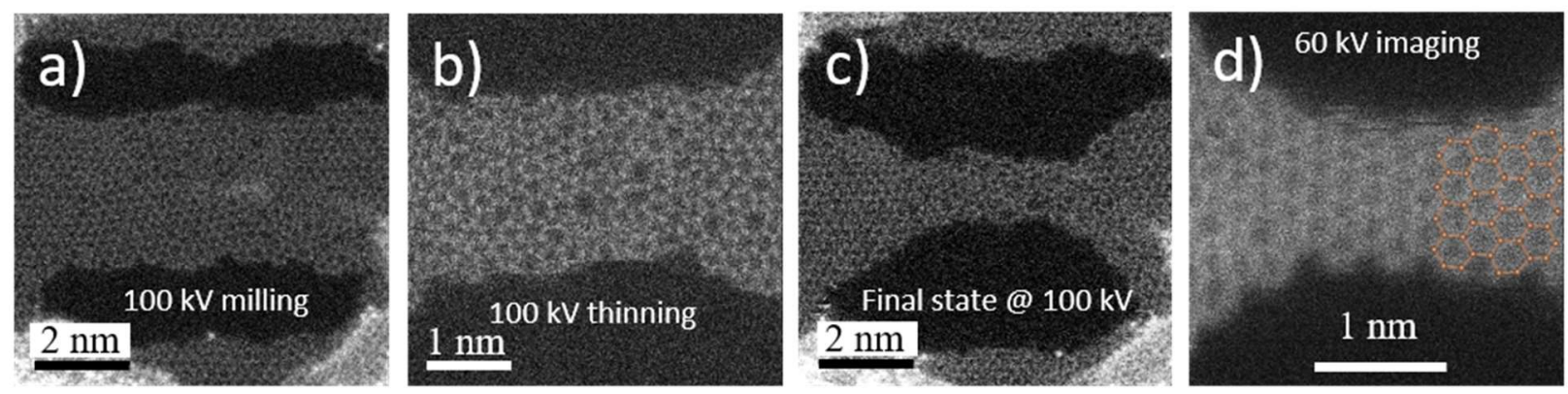

Figure 1. Rapid beam energy change enables fabrication of $1 \mathrm{~nm}$ wide arm-chair graphene nanoribbon during a single, one-day session. a) Milling performed at $100 \mathrm{keV}$ beam energy. b) Nanoribbon further thinned by scanning over central region. c) Final state at $100 \mathrm{keV}$ beam energy. d) After switching to 60 $\mathrm{keV}$ beam energy; gentler beam allows defects to heal and pristine arm-chair terminated section to form. 